Conclusion Chest X-ray is a readily available and commonly performed initial radiological investigation in newborns. Although it can detect most common conditions presenting in newborns, it is important to recognize its limitations and consider further imaging as highlighted in our case series.

\section{MORBIDITY DUE TO ACUTE LOWER RESPIRATORY INFECTIONS (ALRI) IN CHILDREN WITH BIRTH DEFECTS: A TOTAL POPULATION LINKED DATA STUDY}

doi:10.1136/archdischild-2012-302724.0616

K Jama-Alol. Centre for Health Services Research, School of Population Health, The University of Western Australia, Perth, WA, Australia

Background Acute lower respiratory infections (ALRIs) remain the leading cause of hospitalisation among children $<2$ years old. Birth defects occur in $5 \%$ of live births in Western Australia (WA), and are a major cause of childhood morbidity and mortality. However ALRI hospitalisation rates among children with various birth defects are unknown.

Methods We conducted a retrospective cohort study of 245,249 singleton births in WA (1996-2005). Population-based hospital morbidity data and the WA Register of Development Anomalies were linked through the Western Australian Data Linkage System to investigate ALRI hospitalisations in children with and without birth defects. We used negative binomial regression to estimate incidence rate ratios (IRR) for the association between birth defects and number of ALRI hospitalisations over the first 2 years of life, adjusting for known risk factors.

Results Overall $11 \%$ of non-Aboriginal children and $40 \%$ of Aboriginal children with birth defects had a least one ALRI admission before age 2 years. In adjusted analyses, Aboriginal and nonAboriginal children were more likely to be hospitalised for ALRI if they had a birth defect than children with no birth defects (IRR 2.29, 95\% CI: 1.89, 2.78; IRR 2.00, 95\% CI: 1.84, 2.17 respectively). Conclusions WA children $<2$ years with birth defects are at greater risk of morbidity due to ALRIs, when compared to children with no birth defects. Risk of ALRI hospital admission varies between different birth defect categories.

\section{FLEXIBLE BRONCHOSCOPY IN THE ACUTE MANAGEMENT OF CONGENITAL LOBAR EMPHYSEMA}

doi:10.1136/archdischild-2012-302724.0617

1.2ES Abd El-Moneim. 'Pediatrics, Taibah University, Madinah Monawara, Saudi Arabia; ${ }^{2}$ Pediatrics, Sohag University, Sohag, Egypt

Introduction Although uncommon, congenital lobar emphysema (CLE) is a potentially life threatening pulmonary abnormality affecting infants. Lobectomy, sometimes done under emergency conditions, is the universally accepted treatment of CLE with severe symptoms. However, in the developing world trained pediatric thoracic surgeons are not everywhere available. On the other hand, the use of flexible fiberoptic bronchoscopy (FFB) in children has shown an excellent safety record. Furthermore, it can be performed under sedation in most cases. We present here a case of CLE, in which we had been able to relieve the acute respiratory distress using FB.

Case presentation and procedure A distressed 4.5 months female infant was referred to us for repetitive prolonged bronchopneumopathy since birth. Successive chest X-rays showed increasing expansion and hyperlucency of right hemithorax, mediastinal shift, and compression of the left lung. At FFB the apicoposterior segmental bronchus of the right upper lobe (APSB/RUL) was narrow, flaccid and showed an expiratory check valve obstruction. By manipulating and rotating the bronchoscope tip into APSB/RUL and applying suction, we had succeeded to release the trapped air in the RUL. Clinical and radiological manifestations resolved completely following the procedure.

Conclusions In addition to the role it might play in the diagnostic workup of patients with CLE, FFB can be used as a tool for relief of obstruction. In certain situations, this may be life saving, especially in places and settings where emergency lobectomy cannot be arranged.

\section{BENEFIT OF PRENATAL DIAGNOSIS OF COMPLEX CONGENITAL HEART DEFECTS ON NEONATAL MORBIDITY}

doi:10.1136/archdischild-2012-302724.0618

'B Ruijsink, ' $\mathrm{F}$ Landsman, ' $\mathrm{M}$ Slieker, ${ }^{2} \mathrm{D}$ Vijlbrief, ${ }^{1} \mathrm{H}$ ter Heide, ${ }^{3} \mathrm{~W}$ de Boode, ${ }^{4} \mathrm{~K}$ Jansen, 'M Freund. 'Paediatric Cardiology; ' ${ }^{2}$ Neonatology, University Medical Center Utrecht, Utrecht; ${ }^{3}$ Neonatology, University Medical Center St. Radboud, Nijmegen; ${ }^{4}$ Paediatric Intensive Care, University Medical Center Utrecht, Utrecht, The Netherlands

Background and Aims As survival rates in neonatal cardiac surgery improve over time, morbidity becomes more important in complex congenital heart disease (cCHD). We investigated whether prenatal diagnosis of cCHD affects preoperative and early postoperative morbidity in neonates.

Methods Retrospective observational study, performed between July $1^{\text {st }} 2004$ and July $1^{\text {st }} 2011$, including 247 infants with cCHD requiring surgery within the neonatal period. 113/247 patients were diagnosed with cCHD by prenatal screening (PreDx). Morbidity was based on preoperative use of high dose prostaglandin $\mathrm{E}$ and preand postoperative cardiac function, ventilation time, length of admission (LOF), inotropic support requirement and presence of severe complications (resuscitation, acute kidney and liver injury, and neurological events)

Results Complexity of cCHD was higher in children diagnosed PreDx. After correction of complexity, median pre-operative ventilation time ( 3 vs 0 days), LOF (7 vs 9 days) and preoperative cardiac function were significantly better in PreDx neonates; and correction of acidosis, preoperative inotropic support and high dose prostaglandin E was significantly less common. Severe complications occurred significantly less when PreDx. There were no significant differences in postoperative morbidity and overall mortality.

Conclusions Prenatal diagnosis of $\mathrm{CCHD}$ improves pre-operative morbidity significantly. The patient condition is better compared to postDx and the need for preoperative intensive care treatment is less. Seemingly, intensive care treatment can achieve a good preoperative condition, even in neonates deteriorated due to an at birth unknown cCHD. This could explain why post-operative morbidity was equal in both groups.

\section{AN OUTBREAK OF NEURAL TUBE DEFECTS IN IRAQ: A CASE REPORT}

doi:10.1136/archdischild-2012-302724.0619

${ }^{1} \mathrm{~S}$ Dastgiri, ${ }^{2} \mathrm{~F}$ Mahmoudzadeh, ${ }^{2} \mathrm{~A}$ Dastgiri. ${ }^{1}$ Community and Family Medicine; ${ }^{2}$ Tabriz University of Medical Sciences, Tabriz, Iran

Aim The aim of this study was to report an outbreak of Neural Tube Defects (NTDs) in Iraq, and to highlight the possible responsibilities of international and local authorities for an action for the control of this outbreak in this region.

Methods Information for the occurrence of NTDs was gathered from reports published from an Iraqi western region, and for other countries from reports published by the International Clearinghouse for Birth Defects and European Network for Surveillance of Congenital Anomalies.

Results Prevalence rate of NTDs was 33 (per 10,000 births, CI95\%: 21-44) in Iraq while different rates have been reported from various parts of the world ranging from 12.6 (per 10,000 births) in Cuba, 9.6 (per 10,000 births) in Norway, 8.7 (per 10,000 births) in China, 7.03 